\title{
Capacitive Sensing Algorithm for Elderly Activity Detection Scheme Indoor Environment
}

\author{
Atika Arshad, Sheroz Khan, Kushsairy Abdul Kadir, Rumana Tasnim \\ Departement of Electrical and Computer Engineering, International Islamic University Malaysia, \\ P.O. Box 10, 50728 Kuala Lumpur
}

\section{Article Info \\ Article history: \\ Received Feb 11, 2018 \\ Revised Apr 12, 2018 \\ Accepted Apr 28, 2018 \\ Keywords: \\ Capacitive detection \\ Tracking mechanism \\ Elderlies \\ Floor sensing \\ Activity recognition}

\begin{abstract}
The aim of this research is to assist elderlies in accomplishing their everyday tasks through facilitating their home atmosphere with sensors, actuators, and computational resources. A significant effort is needed to incorporate them to make them effective in daily life. Particularly, the tracking and detection of elderly people's daily tasks, associated with the information of the user's location in the home environment signify the key pillars of this work. The detecting and tracking algorithm uses the sensory data to indicate an associated situation in order to allow elderly people with special requirements. Monitoring the elderly's behaviour over a long period of time will allow prediction of their forthcoming alarming situation which allows the finding of the elderly's behavioural deviations in their everyday routine. The proposed resolution has been methodically assessed in the laboratory.
\end{abstract}

Copyright (C) 2018 Institute of Advanced Engineering and Science. All rights reserved.

\section{Corresponding Author:}

Atika Arshad,

Departement of Electrical and Computer Engineering,

International Islamic University Malaysia,

P.O. Box 10, 50728 Kuala Lumpur.

Email: atikaarshad@hotmail.com

\section{INTRODUCTION}

In an elderly home, it is important to know of residents' activities for assigning more support and assistance to them. Monitoring activities such as spending prolonged time in the bedroom, roaming around relentlessly in the house, or unfamiliar absence for a lengthy period of time and so on may be proved effective in knowing about any forthcoming irregular or abnormal happenings. Therefore, it is imperative to propose a conceptual design of a robust system capable of monitoring the activities of elderly homes. In recent years, researchers have developed a variety of health monitoring technologies to assist elderly citizens. A comprehensive literature review in this area was reported by [1-2]. However, before developing a monitoring technique, it is essential to have a better understanding of how to design a monitoring system with requirements suited for elderly individuals’ lifestyles.

This information can be achieved based on the type of sensors needed for monitoring elderly individuals. The sensing categories include; (i) Fall detection, (ii) Activities of everyday monitoring and (iii) Location and position tracking. Fall detection focuses on the occurrence of fall among elderlies, and activity monitoring technique identifies and differentiates everyday activities of a person being in a room or getting on and off the bed in a room and thus detecting irregular conditions. Location and position tracking identify where the person is during an emergency situation. Generally, it is known that elderly individuals are not at ease around new forms of technology. For these reasons, the advancements of unobtrusive techniques and technologies allowing the lengthy monitoring of human beings is a key effort to be researched, particularly in the elderly people's home environment.

Video-based sensing techniques can offer important information about an elderly's current and past actions by posture detection [3]. Nevertheless, this may violate usual need of privacy in the home 
environment [4]. In addition, video processing is an expensive and complicated monitoring method needing more than one camera in a room. It also requires more space and more power. As these devices usually operate as recording devices, they are considered to be privacy violation tools. Another issue for the camerabased system is it is susceptible to shadows as well as reflections and it also has line-of-sight issues.

The acoustic-based system has been found to be a very inconspicuous technology for the purpose of fall detection and activity recognition [5]. Ultrasonic systems are of moderately high cost as such systems consume more power as well as have high complexity issues and also suffer from a good amount of environmental interference. Clothing and textiles easily attenuate the acoustic signals. To overcome this, the ultrasonic transponder should be placed in a visible location. Furthermore, one room needs lots of transducers comprising of extensive cabling and the also user needs to carry a battery-operated transponder all the time.

Generally, PIR (Passive Infrared) sensors are used commonly for monitoring motions. They can be fixed in the ceiling of a room, allowing the evaluation of movements and activities [6-8, 14]. PIR sensors are compact in size, cost-effective, easily installable, less power consuming and can be used as a motiondetecting sensor. Unlike microwave sensor, its waves cannot go through walls. Nevertheless, PIR sensors are comparatively imprecise as they are sensitive to temperature and also cannot monitor motionless or slowly moving human body.

The floor-sensing method is an efficient and potential detection method for future advancements in indoor monitoring. In this method, the monitored person does not require the device to be attached to his body or clothing. The floor sensors are mainly placed underneath the ground or above the ceiling permitting patients to roam around conveniently. Therefore, the floor sensor-based method is the best application for elderly homes as it is inexpensive and portable for activity recognition system [9].

Hence this work proposes a floor sensing device, which can be fitted onto the floor surface, making the system compatible with elderly individuals' existing lifestyles. The proposed system in this work will be able to predict the behavioural pattern of the person. Furthermore, this work is an extension of the work in [10]. The work conducted in [10-11] had discussed tracking of the human body by implementing capacitive proximity sensing technique to sense the existence and movement of the human body. The designed system was proposed to be implemented by installing a matrix of thin planar electrodes underneath the floor surface in the elderly homes, hence, making the system non-invasive and discreet. Parameters studied included mobility and fall. This work further develops a long-term monitoring system where a behavioural profile of the user can be built, which can sense any change in the user's physical condition.

Figure 1 shows the block diagram of the proposed system. The long-term monitoring system comprises of sensors which will enable localization, fall detection and activity recognition. The monitoring sensors are linked to a programmable processing unit. A display unit like a personal computer or a mobile phone is required to store and analyze obtained data.

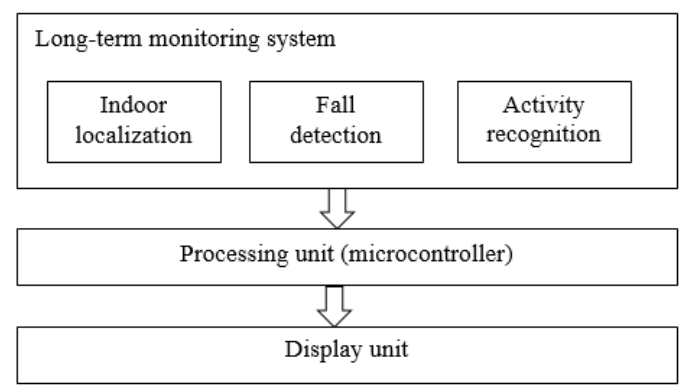

Figure 1. Block diagram of the proposed long-term monitoring system

The key purpose of this work is to assess activity recognition scheme that identifies activities such as sitting on a chair, walking, entering/leaving a room or washroom or going to bed. For sensing any disease of the patient, we must create a behavioural profile of the user. This profile can be carried out by detecting a long-term and repeated behavioural changes from mobility traces. A behavioural profile can sense anomalies to realize probable behavioural deteriorations that can result in urgent conditions related to any diseases.

The proposed system will be able to identify the need for a new generation of non-invasive healthcare systems that make early detection of anomalies and health issues for elderly people by recognizing and detecting their behavioural changes over time. Proper recognition and interpretation of any irregularity and abnormality are crucial for offering a smart healthcare system for the elderly, their family members, and 
caretakers. A healthcare system should offer an extensive usability [12]. Also, it must offer communicative user interfaces and comfortably usable devices for elderly patients. Thus, the proposed system will analyze and recognize the usual nature of elderly person for any incidence of anomalies in their regular behaviours and detect behavioural nature of the person. Such proficient and complete monitoring technique will ensure independent healthcare with less hospitalization.

\section{RESEARCH METHOD}

Capacitive sensors have the prospect to recognize activity as it is an unobtrusive system and does not necessitate any monitoring device all the time. The floor sensing system proposed consists of a network of capacitive proximity sensor plates embedded under the floor seamlessly, for human localization and tracking which is also capable of supporting fall algorithms for monitoring falls. For each sensor plate forming the floor, it can detect the occupancy information, whether a person is present or absent to indicate and track the movement of a person from one place of a room to another, approximate the location of the person by using threshold mode. The system starts with the sensing and data acquisition where a microcontroller is used for data processing. The microcontroller tests the occupancy of a sensor plate by deciding the threshold value. When the signal changes into a different value from threshold level, an object's existence can be understood. When the signal comes back under this threshold level, it is understood that the object was disengaged from his body. When an elderly person shifts within the room, the localization algorithm can follow the location of the body. Due to the movement of the patient, the value of capacitance (threshold value) of the sensor varies in response to their distance to the body in this way the elderly's daily movement pattern can be extracted.

The floor sensor is built on a network of 25 capacitive sensors, each sensor is having a square size of $60 \mathrm{~cm}$ by $60 \mathrm{~cm}$, and large enough to assure an average foot is dependably sensed. The sensors are placed on the floor surface with a $5 \mathrm{~cm}$ gap between the sensors. Furthermore, the sensors are located in a 5 x 5 matrix. x11 symbolizes the coordinate position of the first sensor for row 1 and column 1 of the floor sensing tile, and in that order, x55 symbolizes sensor 25 with the matrix arrangement of row 5 and column 5 of the floor tile. A plan of the floor placement system and the sensing and processing system is displayed in Figure 2 and Table 1 displays the hardware pin connection with the microcontroller unit. For the notification of any sudden falling occurrence or deteriorating physical conditions of the elderlies, a buzzer is used to inform the caretakers or family members of accidental scenarios and occupancy status information.

Table 1. The hardware pin connection with the microcontroller unit

\begin{tabular}{lll}
\hline Assigned MCU Pin Number & Component & Device \\
\hline D22-D46 & Capacitive sheet & Floor sensors (25 sensors) \\
D13 (PWM) send pin & Capacitive sheet & Capacitive sensors \\
D47 & Alarm & Buzzer \\
\hline
\end{tabular}

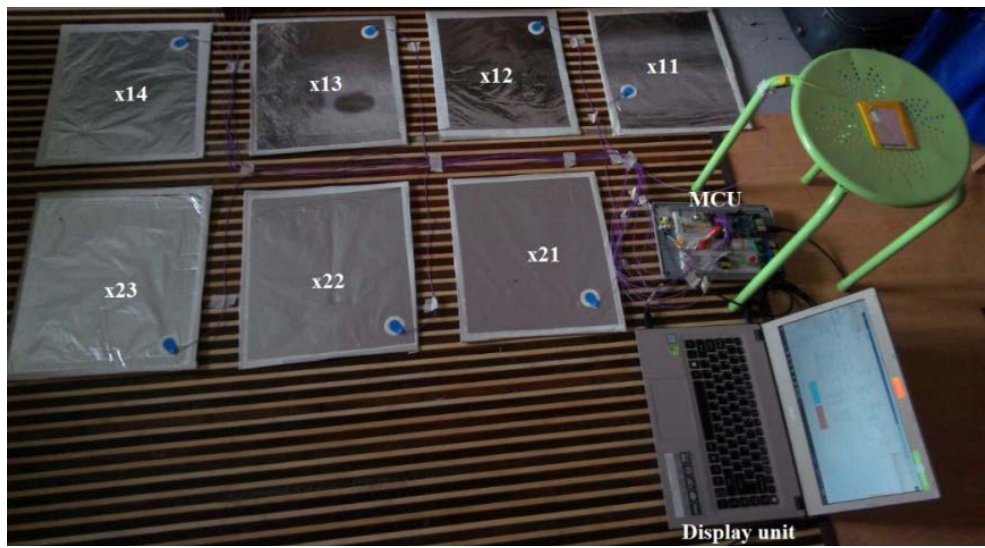

Figure 2. Experimental setup of the floor monitoring system [10]

The capacitance variation can be measured by achieving sensor readings and any changes as well. Then sensor readings sense any changes that go from an exact threshold value. Also, the occupancy status can be analyzed within a time period and relate them to some activity level. 
Detection of human activities is based on the features obtained for motion analysis. Inactivity recognition system, any variation in capacitance value due to the movements of the user is given by the MCU. In order to monitor the activity such as leaving/entering a washroom, going to bed and so on. In this experimental work, it was assumed that the sensor x11 was placed at the entrance of the toilet, x12 was placed at the entrance of the bedroom and x13 was placed near to the bed. A counter was connected to these specific sensors. This acted like a data storage unit, which will count the number of times each sensor was occupied with respect to time. Hence, elderly behaviour can be documented based on the sensor coordination position, (active or inactive).

Figure 3 shows the algorithm to determine the occupancy state of the sensor plate. In this experiment, the threshold value is about 6000 . These sensor reading values are a long integer including the capacitance, in arbitrary units (a.u.). When the sensor reading becomes 6000 or more, a sensor plate is understood to be occupied.

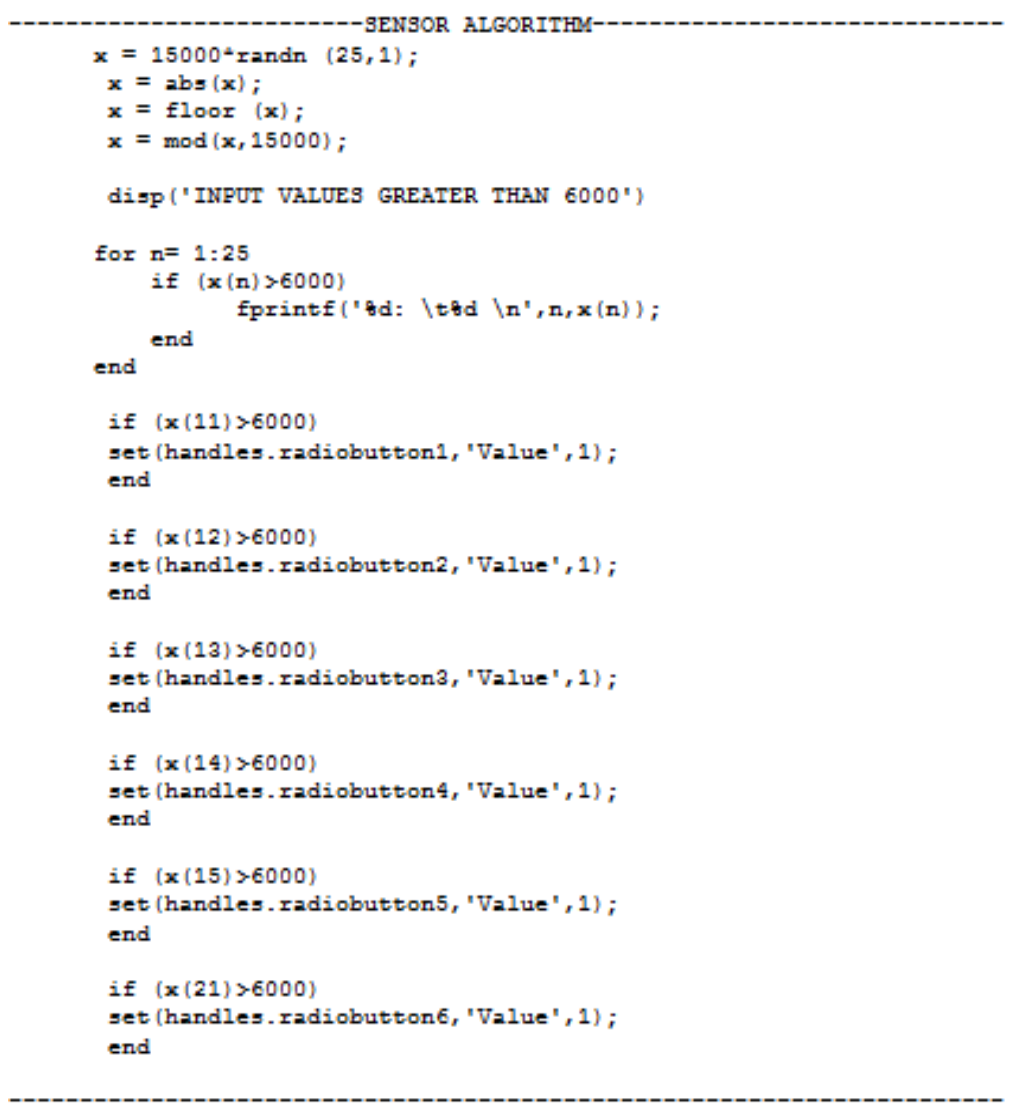

Figure 3. Sample of the programming algorithm to determine the occupancy state of the sensor plates

\section{RESULTS AND ANALYSIS}

In this work, the results obtained are visualized using MATLAB. The results are obtained will show the occupancy of the sensor plates and the activity recognition. Figure 4 illustrates the occupancy of a single sensor, over a period of five minutes. The x-axis of the graph represents the time and the y-axis represents the sensor threshold value in arbitrary unit. It can be observed from the figure that in the beginning of the time, the signal peak is very low, showing that no human is occupying the sensor. After some time the peak reaches to a maximum, followed by a drop of the signal waveform. The maximum peak attained illustrates the occupancy of the sensor, it can be noted that the peak has a threshold value more than 6000 . It can also be noted that there are smaller peaks being formed beside the main big peak. The small peaks are the sensor readings from the neighbouring sensors since the sensors are proximity sensors the nearby sensors are able to sense the presence of a conductive body within a certain range. It is noteworthy to observe the fall of the threshold after some time, this drop is due to the person leaving the sensor and when the person has left from that sensor, the signal begins to fade away. 
Figure 5 shows the graph when a person stands on two sensors. Two maximum peak is available in the figure since the person occupies the two sensors positioned at x11 and x21. The two peaks represent the contact of the sensor with the person. Similarly, for every occupancy of a senor, the occupancy state can be represented graphically. In this way, a movement profile can be built.

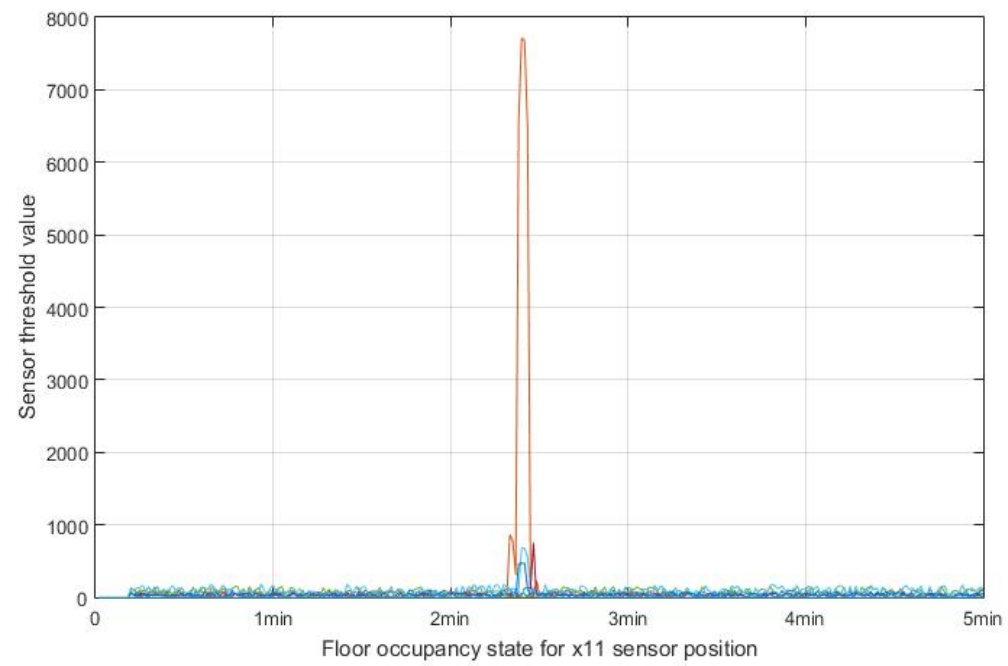

Figure 4. Graphical representation of the floor occupancy state for x11 sensor position

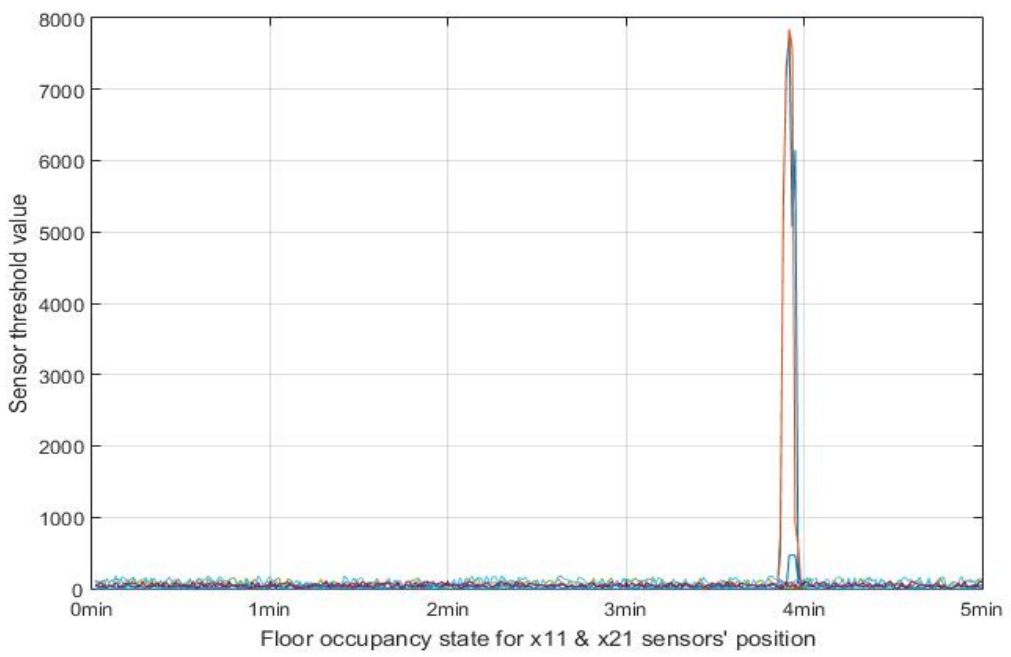

Figure 5. Graphical representation of the floor occupancy state for x11 and x21 sensor position

Figure 6 indicates the frequency of usage of the toilet over a period of a day. Data pattern can assist with the detecting of urinary tract infections through the frequency of bathroom visits throughout the day. Take a case scenario, a healthy elderly may use the toilet 10 times in a day, this activity is assumed to be a fixed number of times per unit duration for a healthy condition. For the same activity during the same unit duration if the toilet sensor $\mathrm{x} 11$ is activated more or less than the healthy condition. From this, the deteriorating situation can be noticed and prediction can be made on their deteriorating health condition, thus allowing the caretakers to take action before resident health worsens further. 


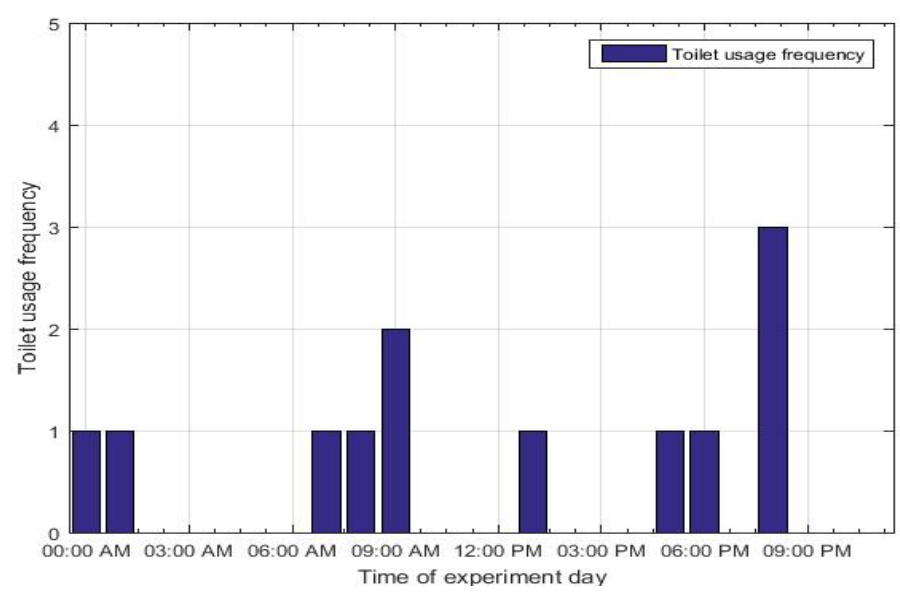

Figure 6. Demonstrates the frequency of toilet usage in a day

This service permits the forecast of the elderly's activities around the clock, hence allowing the system to learn the various patterns so that decisions can be made on their wellness level.

\section{CONCLUSION}

The approach established in this work has the objective to develop a tracking system that would aid in predicting the wellness of a person performing daily activities over a long term. The long-term monitoring of human well being can prevent the sudden sickness of elderly people. Due to these reasons, the advancement of long-term tracking methods of elderly good beings needs to be worked on, particularly in the elderly homes scenario. The proposed system can sense any behavioural changes of the regular actions based on a generic indoor localization technique and a swarm intelligence method. This will permit the family members or caregivers in analysing the behavioural changes of daily activities of elderly. Capacitive proximity sensing was chosen for the development of this work as it is a prevailing technology nowadays that is capable of being discreetly integrated into a range of applications. Here, capacitive floor sensors are implemented as occupancy sensors.

\section{ACKNOWLEDGEMENT}

Financial assistance for this research by the IIUM Research Management Center (RMC) via RIGS Grant No RIGS 15-147-0147 is highly acknowledged.

\section{REFERENCES}

[1] Arshad, A., Alam, A. H. M. Z., Khan, S., Tasnim, R. Passive monitoring techniques for elderly: a review of recent development and prospective. Proceedings of the World Congress on Engineering and Computer Science. 2015; 1.

[2] Arshad, A., Khan, S., Zahirul Alam, A., Ismail Ahmad, F., Tasnim, R. A Study on Health Monitoring System: Recent Advancements. IIUM Engineering Journal. 2014; 15(2); 87-99.

[3] Jalal, A., Kim, Y. H., Kim, Y. J., Kamal, S., Kim, D. Robust human activity recognition from depth video using spatiotemporal multi-fused features. Pattern recognition. 2017; 61, 295-308.

[4] Alcala, J.M., Urera, J., Hernandez, A., Gualda, G. Assessing Human Activity in Elderly People Using NonIntrusive Load Monitoring. Sensors 2017. 2017; 17 (351); 2-17.

[5] Pattamaset, S., Charoenpong, T., Charoenpong, P., Chianrabutra, C. Human fall detection by using the body vector. 9th International Conference on Knowledge and Smart Technology (KST). 2017; 162-165.

[6] Popescu, M., Hotrabhavananda, B., Moore, M., Skubic, M. VAMPIR- An Automatic Fall Detection System Using a Vertical PIR Sensor Array. 6th International Conference on Pervasive Computing Technologies for Healthcare. 2012; 163-166.

[7] Al-Naimi, I., Wong, C. B. Indoor human detection and tracking using the advanced smart floor. 8th IEEE conference on Information and Communication Systems (ICICS). 2017; 34-39.

[8] Al-Naimi, I., Wong, C. B., Moore, P., Chen, X. Multimodal approach for non-tagged indoor identification and tracking using smart floor and pyroelectric infrared sensors. International Journal of Computational Science and Engineering. 2017; 14(1); 1-15. 
[9] Ranasinghe, S., Al Machot, F., Mayr, H. C. A review on applications of activity recognition systems with regard to performance and evaluation. International Journal of Distributed Sensor Networks. 2016; 12(8); 1550147716665520.

[10] Arshad, A., Kadir, K. A., Khan, S., Ismail, A. F., A.H.M Alam, Z., Yusof, Z. M. Capacitive proximity floor sensing system for elderly tracking and fall detection. 4th IEEE conference on Smart Instrumentation, Measurement and Applications (ICSIMA). 2017.

[11] Eni Folendra Rosa, "Affective Disorders in The Elderly: The Risk of Sleep Disorders," IJPHS, Vol. 7, No. 1, March 2018

[12] Banos, O., Villalonga, C., Bang, J., Hur, T., Kang, D., Park, S., \& Hong, C. S. Human behavior analysis by means of multimodal context mining. Sensors. 2016; 16(8); 1264.

[13] Atika Arshad, Sheroz Khan, Kushsairy Abdul Kadir, Rumana Tasnim, "An Activity Detection scheme of Elderly Behaviour at Room Environment Using Capacitive Sensing Algorithm," Proceedings of the IEEE International Conference on Smart Instrumentation, Measurement and Application (ICSIMA 2017), 27-28Nov2017, The Everly Hotel Putrajaya, Malaysia.

[14] T.S. Gunawan, I.R.H. Yaldi, M. Kartiwi and H. Mansor, "Performance Evaluation of Smart Home System using Internet of Things,” International Journal of Electrical and Computer Engineering (IJECE), 8(1), pp.400-411, 2018. 